Manuscript

\title{
An alternative technique for ultra-high resolution bathymetry and seabed inspection for marine renewables
}

\author{
Francisco Francisco, Jan Sundberg* \\ ${ }^{*}$ Division of Electricity, Uppsala University, Box 534, 75121 Uppsala - Sweden \\ franciscogemo@gmail.com; Jan.Sundberg@angstrom.uu.se
}

\begin{abstract}
Marine renewable energy technologies have a great potential in supplying clean electricity to millions of people across the globe, if technical and economic conditions are in right. So far, ocean energy projects are commonly started by SMEs or educational institutions with limited budgets. Therefore, any effort to reduce expenses is of great value. One of the areas involving substantial expenses are the inevitable seabed inspection prior to deployment of marine renewable energy device. Detailed seabed inspections can also reduce the risk of associated with deployment of structures on uneven seabed, especially marine renewable energy devices with gravity foundations. By reducing the costs and risks of such surveys prior and during the installation phases, the feasibility of marine renewable energy projects can be more favoured and competitive. In this perspective, this study proposes a cost and time effective technique for seabed surveys. The proposed technique involves the use of high precision and inexpensive sonar systems and underwater optical cameras integrated into a versatile and compact subsea monitoring platform. It also involves simple and practical data acquisition and processing protocols that do not requires hi expertise for operation. The results obtained showed that high resolution bathymetric maps and detailed seabed inspections imagery can be acquired. This study concludes that a simple and inexpensive subsea monitoring platform comprising a multibeam, dual beam and video cameras can be effective for high resolution seabed inspection and bathymetric measurements for marine energy applications.
\end{abstract}

Keywords: Bathymetry, marine renewables, seabed inspection, high resolution survey, sonar

\section{Introduction}

The future of the world's energy security depend on a successful implementation of renewable energy solutions that not only are emission-free, but most importantly economically attractive, safe and reliable. There is an extraordinarily high potential for the marine renewable energy sector in terms of source availability, energy conversion and commercialization. However, working in offshore environments involve higher costs and technical challenges on every aspect and phase of projects. Detailed marine surveys to evaluate the seabed conditions prior to deployment of marine renewable energy devices is an indispensable task. Seabed surveys and environmental monitoring are standard requirement needed for permitting and consenting marine energy projects.

Among several physical environmental aspects, the characteristics of the energy resource, bathymetry and seabed are three parameters playing important roles in the feasibility of marine renewable energy projects. The characteristics of the energy conversion devices themselves also dictate the location where deployment should take place. For example, marine renewable energy devices are either floating, bottom fixed or onshore fixed 
platforms [1]-[3]. Regarding the type of foundation to be utilized, the most common are the monopole, gravity foundation, tripod foundation, and jacket foundation [4], [5]. In all cases, a bathymetry survey and seabed inspections are needed before the deployment of marine renewable energy devices. Commonly these surveys begins with a geophysical and geotechnical seabed inspections that are undertaken with a great precision and accuracy in order to gather information about the depth, benthic and subsoil composition, as well as identification of a variety objects lying on the seabed that may pose risks for future deployments. These surveys normally involve substantial costs, time, human resources, instrumentation, and large vessels. Apart from that, these surveys have complex procedures and preconditions that not always can be met, especially in remote locations or in poor energy markets worldwide. Conventional survey methods are effective for mega projects, when large areas need to be covered and when the costs of the survey are easily capped by large investors. However, in reality, most of the important future markets for marine renewables, especially wave power, may be developing countries or isolated islands aiming for smaller projects and with limited budgets. Therefore, large costs of surveys, cabling, logistics, etc, may hamper potential investors. Alternative survey techniques with low costs may therefore revitalise the marine renewable energy sector. Such techniques would simplify survey procedures, reduce the amount of human resources while delivering acceptable results.

This study aims to introduce an efficient technique of surveying the seabed and for gathering high resolution bathymetric data. This include the use of small vessels with very limited personnel (on average two), multibeam imaging sonar (MBS), dual beam sonar (DBS), and underwater cameras (UWC), all coupled with the global positioning system (GPS). This study used the Uppsala University wave power converter (UU-WEC) [6], [7] as an example. The most relevant physical variable for the deployment UU-WECs is the bathymetry which needs to be in adequate range of $20 \mathrm{~m}$ to $100 \mathrm{~m}$ of depth.

\section{The survey technique}

A simplified technique for evaluating the bathymetry and seabed conditions tested in this study comprise a hardware setup, survey design and data processing algorithm. The platform included a portable mounting system consisting of a pole mount with an adjustable length of $1 \mathrm{~m}-5 \mathrm{~m}$, and a tripod that could be lowered to the seabed. These platforms contained a MBS, a DBS, and UWCs (Table 1). The pole mount was made of lightweight aluminum tube attached to a thin and lightweight aluminum baseplate that houses the measurement instruments (Figure 1a). The same baseplate could be reattached to form a tripod (Figure 1b). The instruments were installed at a specific yaw $(\theta)$, pitch $(\beta)$ and roll $(\gamma)$ angles, and were deployable at controlled drafts (Ds), through scales put on the pole and rope. The baseplate containing the instruments was designed to act as a hydrodynamic stabilizer. Two underwater cameras were used, one (UWC1) aligned with the field of view (FOV) of the MBS and other (UWC2) aligned with the DBS. A GPS module should be properly coupled to the platform, and its reference height known. The installation of the GPS modules is important to assure accuracy of the measurements, because they provide instantaneous geographic location and heave readings. 
Each instrument has its own data processing protocol. The data from the MBS was acquired as a sequence of 768 beams forming an acoustic image containing the backscatter intensity, range, and positioning. Data was pre-processed using a BlueView - SDK 3.6 and ProViwer4 software. The resulting acoustic images were analyzed using Visual Studio and Matlab [8]. The DBS acquired data of acoustic backscatter intensity and range of targets within the acoustic beam path. Each ping comprises of two bifocal beams that provides acoustic backscattering intensity and positioning data among other parameters. DBS data was processed and analyzed using Sonar Viewer and Matlab [9], [10]. The UWCs have a simple and well known data acquisition and processing protocol that can be enhanced using Matlab.

Table 1. Technical specifications of the instruments integrated to the survey platform

\begin{tabular}{|c|c|c|c|}
\hline Component & Specification & Component & Specification \\
\hline $\begin{array}{l}\text { MBS } \\
\text { BlueView }\end{array}$ & $\begin{array}{l}\text { Frequency: } 0.9 \mathrm{MHz} \text { (operational) } \\
\text { Number of Beams: } 768 \\
\text { fps: up to } 50 \mathrm{~Hz} \text { (sample frequency) } \\
\text { FOV: } 132 \times 20 \text { (field of view) } \\
\text { Resolution: } 0.18^{\circ} / 2.54 \mathrm{~cm} \\
\text { Maximum range: } 100 \mathrm{~m}\end{array}$ & \begin{tabular}{|l|} 
DBS \\
Laurent HDS \\
$5 x-G e n 2$
\end{tabular} & $\begin{array}{l}\text { Frequency: } 50 / 200 \mathrm{KHz} \text { (operational) } \\
\text { Number of Beams: } 2 \\
\text { FOV: } 29^{\circ} \text { | } 12^{\circ} \\
\text { Maximum range: } 762 \mathrm{~m}\end{array}$ \\
\hline Component & Specification & Component & Specification \\
\hline $\begin{array}{l}\text { UWC } 1 \\
\text { Mobotix G25 }\end{array}$ & $\begin{array}{l}\text { Lense: } 12 \mathrm{~mm} / \mathrm{F} 2.0 \\
\text { FOV: Hemispherical }\left(180^{\circ} \text { x } 160^{\circ}\right) \\
\text { Sensitivity: 0,05 lux } \\
\text { Max. image resolution: } 2048 \text { x } 1536(5 \mathrm{MP}) \\
\text { Max frame rate: (M-JPEVGA: } 22 \mathrm{fps} \\
\text { Video stream: (MxPEG) } 30 \mathrm{fps}\end{array}$ & $\begin{array}{l}\text { UWC } 2 \\
\text { Axis 2880CS }\end{array}$ & $\begin{array}{l}\text { Lense: } 2.8-8 \mathrm{~mm} / \mathrm{F} 1.2 \\
\text { FOV: } 92^{\circ}-32^{\circ} \\
\text { Sensitivity: } 0.04 \text { lux } \\
\text { Max. image resolution: } 2592 \times 1944 \text { (5 MP) } \\
\text { Max frame rate (M-JPEG): } 30 \mathrm{fps} \\
\text { Video stream (MxPEG): H.264 }\end{array}$ \\
\hline
\end{tabular}

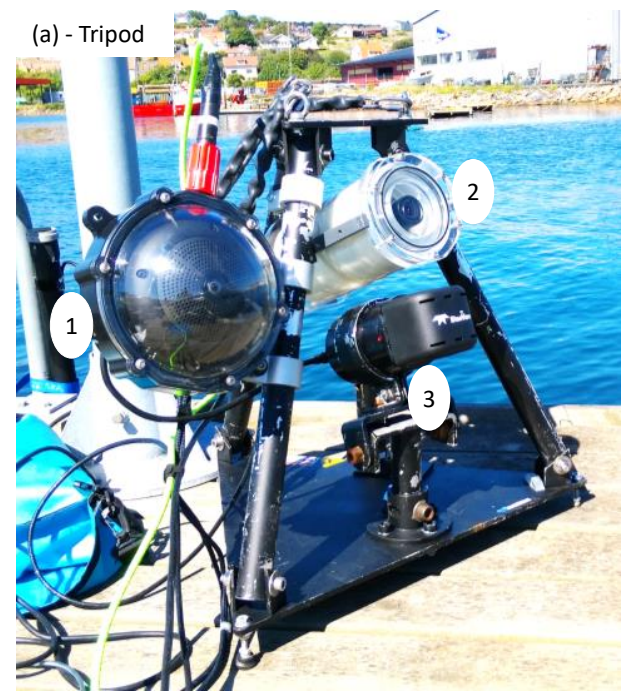

(b) - Pole mount

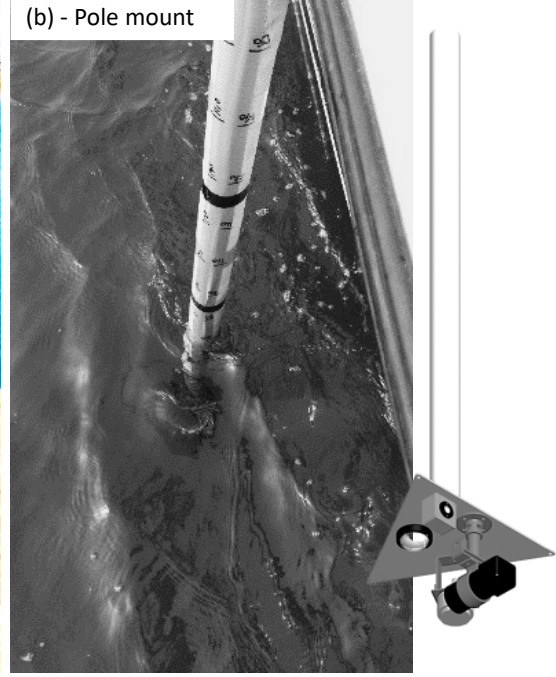

Figure 1. A tripod and a pole mount with measurement instruments (1-2) underwater cameras UWC1-2 and (3) multibeam sonar integrated into a platform for bathymetry and seabed inspection.

\subsection{The survey design}

The surveys can be split in two or more stages, using adaptive outline and detailed outline transects. Outline scouting combines the systematic zig-zag with intensive parallel transects in order to maximize coverage and minimize time (Figure 2a). Intensive scouting utilize systematic parallel transects in order to maximize de spatial resolution (Figure $2 b$ ). The systematic zig-zag and parallel transects are useful to measure depth near the coastline [10]. 


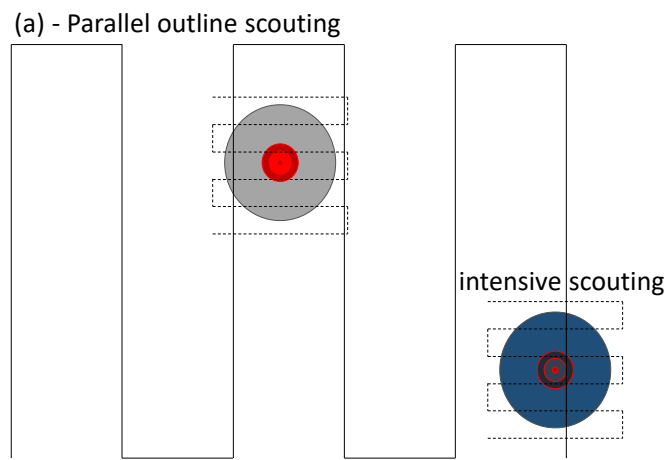

(b) - Zig zag outline scouting

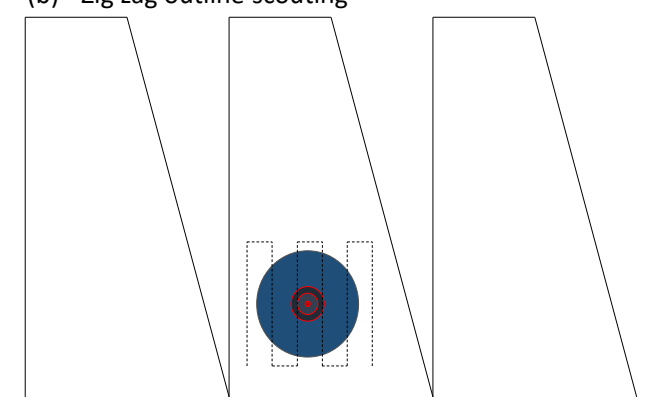

Figure 2. Illustration of a two-stage sistematic scouting survey adopted to gather bathymetry data and seabed inspenctions. (a) combination of outline zig-zag with intensive parallel transects for maxim efficiency; (b) combination of outline and intensive parallel transects for maximum resolution. The lines are the tracks, and circles represent targets, or focused areas.

Among several conducted surveys to support the deployment and operation of UUWECs and similar WECs [11], this article presents three examples of bathymetric and seabed inspection surveys. A combination of systematic zig-zag and parallel transects were used for bathymetric measurements, and parallel scouting and intensive transects were used for seabed inspection. The MBS and DBS were pole mounted and deployed with different drafts $\left(Z_{\text {draft }}\right)$ in the interval between $0.1 \mathrm{~m}$ to $4.0 \mathrm{~m}$ (configuration (a), Figure 3), the MBS and WUCs were fixed to a tripod and deployed down towards the seabed (configuration $(b)$, Figure 3). The MBS was deployed in three different pitch angles $\left(\beta_{M B S}=10^{\circ}\left|45^{\circ}\right| 90^{\circ}\right)$ in relation to the water level. The DBS was always deployed with a constant pitch angle of $90^{\circ}$. The camera were deployed with pitch angles of $10^{\circ}\left|45^{\circ}\right| 90^{\circ}$. All the measurement instruments were put to sample continuously at maximum sampling frequency.

The surveys were conducted from onboard of a small-size vessel (ca. $8 \mathrm{~m}$ long) and the data was collected in phases (Figure 3). First, the survey vessel scanned the overall area using scouting transects to access the general bottom inclination and locate hazard objects on the seabed. During this phase, the vessel traveled at moderated velocity of ca. 5.4 knots, and data gathered was used to pinpoint the locations that required higher resolution measurements of both bathymetry and seabed inspection. The second phase of the survey aimed to gather higher resolution measurements. Here the vessel traveled at constant velocity not exceeding 3.3 knots, while conducting intensive transects over the focal areas identified in the first phase of the survey. 


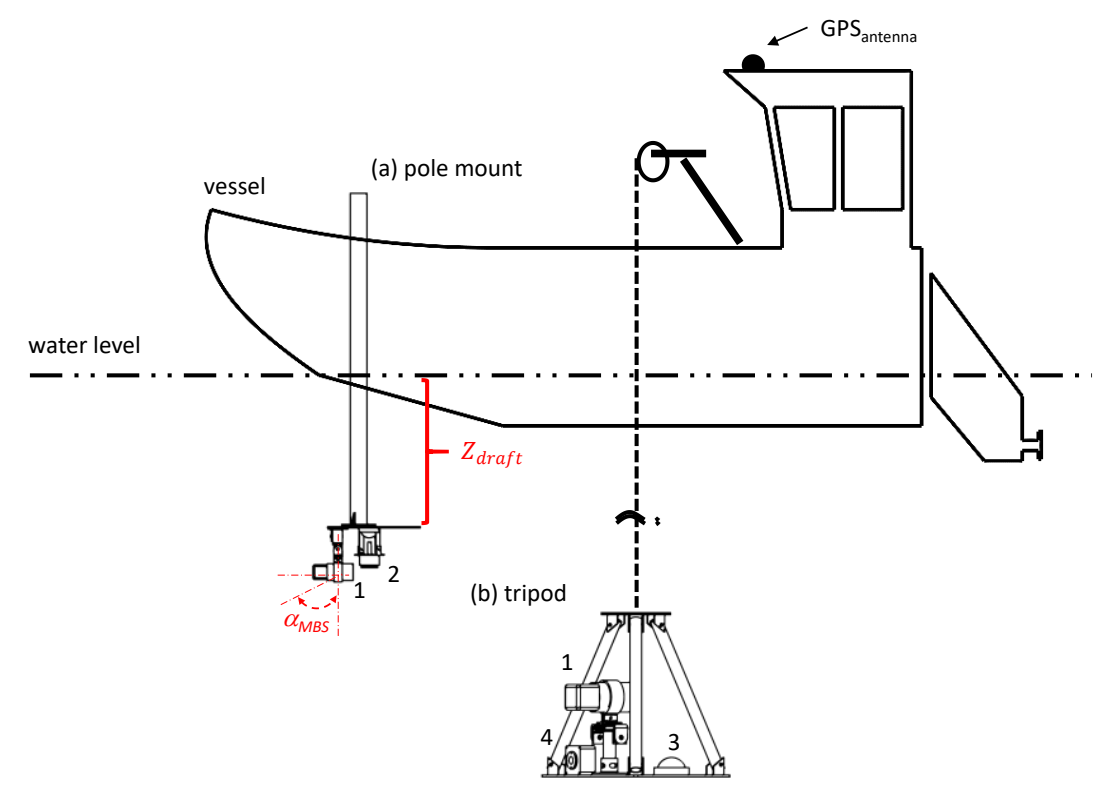

Figure 3. Diagram showing how the instruments were attached to the surveying vessel. On the (a) pole mount were the multbeam (1) and dual beam sonar (2), on the (b) tripod were the multibeam sonar (1) and underwater cameras (3-4).

The spatial coverage had to be the highest possible within the focused areas, and the survey duration had to be short, as this is the ultimate aim of this practice. The bathymetric measurements were done using the DBS with beam width $(\phi)$ of $12^{\circ}$. The spacing between tracks $\left(W_{T}\right)$ was obtained using the sonar's footprint or swath $\left(a_{D B S}\right)$ which was the instantaneous area of the seabed covered by the acoustic beam, as a function of depth $(Z)$ and beam width $(\phi)$ (Equation 1). The beam width was known, therefore, Equation 1 can be rewritten and the spacing between transects known (Equation 2). The spatial resolution $(r)$ was estimated using Equation 3, inspired by the degree of coverage introduced by [12]. The degree of coverage is a survey effort estimated for fish and biomass, it only considered the total track length $(D)$, and total survey area $(A)$. While the newly introduced Equation 3 also includes the spacing between tracks $\left(W_{T}\right)$ and the minimum distance between measured points taking into account the depth, the sonar operating frequency $(\mathrm{f}=200 \mathrm{kHz})$ and speed of sound $(v \cong 1500 \mathrm{~m} / \mathrm{s})$.

$a_{D B S}=2 \cdot Z \cdot \tan \left(\frac{\phi}{2}\right)=2 \cdot Z \cdot \tan \left(\frac{12}{2}\right)=(-0.6) \cdot Z \quad$ Equation 1

$W_{T}=\int 0.6 \cdot Z \cdot d z \quad$ Equation 2

$r=\frac{W_{T} \cdot D}{\sqrt{A}}+Z \frac{v}{f} \quad$ Equation 3

With a high precision sonar, the common source of depth measurement errors would mainly be the controllable and uncontrollable false echoes. Uncontrollable false echoes are caused by targets other than the seabed that can be fish, kelp, soft sediments, or water layers with different densities [13]. Controllable false echoes results from the relative motion between the vessel and the seabed that the sonar cannot tolerate, for example: excessive cruising velocity, heave, or side echoes caused by steep pitch and roll angles [13]-[15].

In our case, the DBS was set to the shortest pulse length (ca. $140 \mathrm{~ms}$ ) in order to maximize the space-time resolutions and improve the measurement accuracy. The pitch, 
roll and azimuth offsets were re-calibrated before each survey by starting the DBS at a sheltered location where the water was more or less still. Operating frequency of $200 \mathrm{kHz}$ was used in order to minimize the uncontrollable false echoes. This set of measures aided the signal processor learning to differentiate correct depth echoes from false echoes. The acquired bathymetric data was analyzed using a simplified algorithm described in Equation 4 assuming that errors caused by pitch and roll were small enough to be and auto-corrected by the sonar computer. The module of the instantaneous measured depth $(Z)$ was calculated taking into consideration vertical offsets caused by heave, tide and draft of the transducers.

$Z=z_{\text {eco }} \pm z_{\text {heave }}-z_{\text {tide }}+z_{\text {draft }} \quad$ Equation 4

where $z_{\text {eco }}$ is the instantaneous height of the sonar in relation to the seabed, $z_{\text {heave }}=$ $\frac{1}{2} \sqrt{s t d\left(z_{\text {eco }}\right)^{2}+s t d(h)^{2}}$ is heave component derived from instantaneous height $(h)$ measured by the GPS, $z_{\text {tide }}$ is the actual height of the tide, and $z_{\text {draft }}$ is the draft of the transducers.

The actual depth over a point (longitude, latitude, $Z$ ) of the seabed is given by the mean instantaneous depth $\bar{Z}$ over a short period of time $\Delta t$. In other words, $t$ is equivalent to the duration of a set of individual pings $(n)$, each with depth value $Z=Z_{t}$, as formulated in Equation 5. High resolution maps were constructed from the matrix $\boldsymbol{H}$ containing all the measured depth points (longitude, latitude, Z), using pseudo-colour plot in Matlab [16]. The pseudo-colour plot comprises a rectangular array of cells with colours determined by value of $Z$. Each cell is made of four adjacent points, and the colour map is formed by linear interpolation of each cell across the entire range of $Z$.

$$
\bar{Z}=\frac{\sum_{i=t}^{n} Z_{t}}{n} \quad \text { Equation } 5
$$

There were sources of errors, and quality control techniques that needed to be addressed for a good depth measurement survey. Errors in depth determination could be divided into three types: large errors or blunders, systematic errors and random errors [13], [17], [18]. Blunder are errors made by the instruments due to defective mechanical or electronic components. Systematic errors were mainly the result of the offsets (fixed errors) or biases (errors that vary under different conditions) in motion sensing of the survey vessel, misalignment of the transducer and other sensor mounting angles. Such errors can easily be corrected if the sign and size of the systematic error can be identified. This category of errors can be determined and removed during installation and calibration of the Sonar survey system. After removing blunders and systematic errors in the depth data, random errors will remain and these can be analysed using statistical techniques. This study considered the following standard errors: errors due to bottom slope (automatically corrected by the sonar); errors due to roll and pitch, error due to heave; error due to draught or draft; and error due to squat. The estimated drift (error) on the corrected depth, at the $68 \%$ confidence level, was obtained using the equation: $\sigma_{Z}=\sqrt{\sigma_{\text {Zdraft }}+\sigma_{\text {Zsquat }}+\sigma_{\text {Zheave }}+\sigma_{\text {Ztide }}}$ [13], [8], [9]. 


\section{Examples of bathymetric measurements and seabed inspection}

A number of surveys were conducted using a MBS coupled with DBS and UWCs to measure depth and access the seabed conditions to support the deployment of WECs. Seabed inspections were also conducted to evaluate water pathways for large deployment vessels that needed to dock in narrow and shallow harbors where WECs are loaded and unloaded. As examples, three surveyed locations are presented in this study: a wave power test site in Lysekil, Sweden (Example 1) where more than ten WECs have been deployed; a small harbor beside a WEC factory in Lysekil (Example 2); and a proposed wave power site in Adah, Ghana (Example 3).

\subsection{Example 1}

The seabed in the Lysekil wave power test site is flat and has in average $25 \mathrm{~m}$ of depth (Figure 4). Here the surveys were conducted using intensive parallel scouting transects (Figure 5a). The DBS data extracted from one of transects show an average depth of approximately $25 \mathrm{~m}$ (Figure $5 \mathrm{~b}-\mathrm{c}$ ). These surveys were conducted with $W_{T}$ of ca. $15 \mathrm{~m}$, at spatial resolution $r$ of ca. $0.3 \mathrm{~m}$.

Seabed inspections confirmed that the seabed inside the test site is composed soft substrate, mostly sand (Figure $5 \mathrm{~d}$, e). However, underneath the sand are hard substrates that rises to form rocky islets such as indicated as $i i$ in Figure 4 and shown on the acoustic image in Figure 5f. Structural inspections were also conducted, and the results show acoustic images of WECs and its surroundings (Figure 5e).

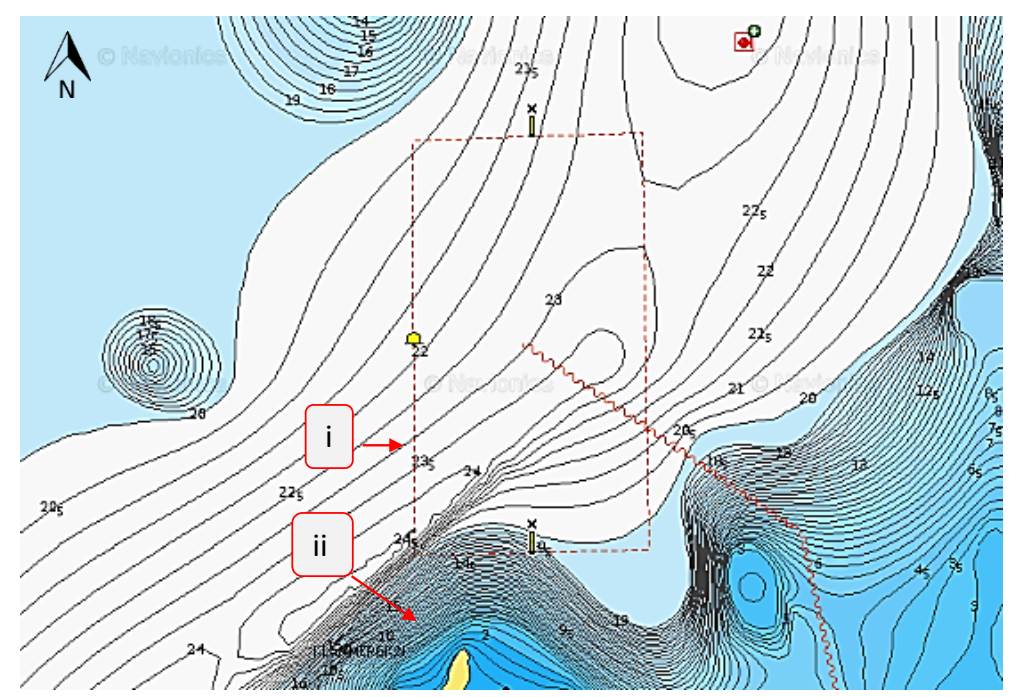

Figure 4. Overview of the bathymetry on in the Lysekil wave power test site showing bottom depth values of $23 \mathrm{~m}-25 \mathrm{~m}$. The perimeter of the site is demarked with the red dashed line (i), and a nearby rocky islets (ii). 

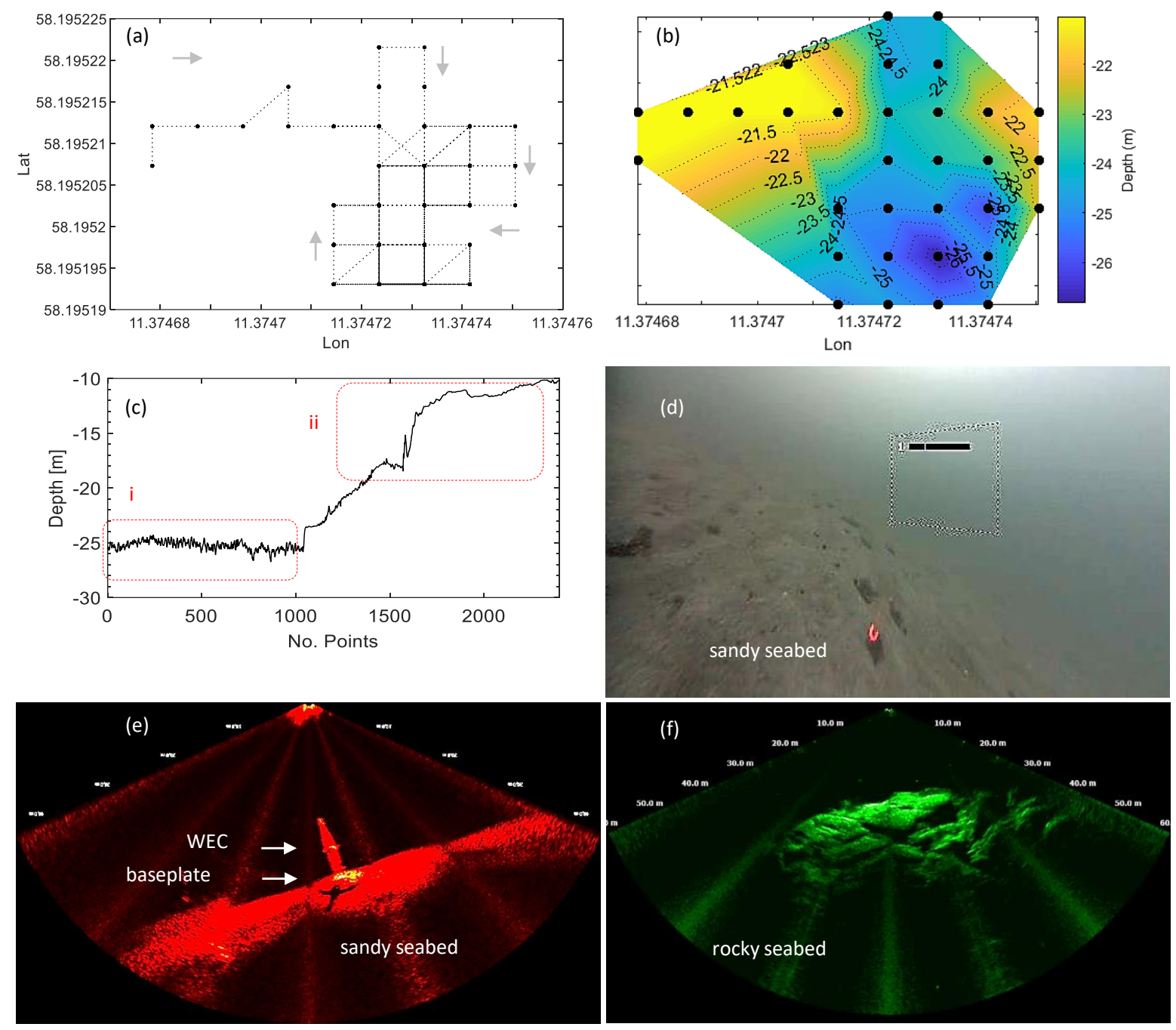

Figure 5. Example of ultra-high resolution bathymetry, seabed and structural inspections in the Lysekil test site. (a) Intensive parallel scouting survey transects; (b) A view of a ultra-high resolution bathymetric map; (c) Raw data belonging to one of surveyed transects i: sandy seabed, and ii: rocky islet. (d) Optical image of the seabed acquired suing a UWC. (e, f) Acoustic images showing: (e) wave power converter deployed on the sandy and flat seabed of the test site (i); (f) The rocky seabed of the nearby islet indicated as ii in Figures 4 and 5c.

\subsection{Example 2}

A harbor located in Lysekil, close to a WEC factory, was also surveyed in order to evaluate if depth was enough for navigation of a large offshore deployment vessel (Figure 6). Here the survey was conducted using a $W_{T}$ of ca. $3 \mathrm{~m}$, resulting on a spatial resolution $r$ of ca. $0.075 \mathrm{~m}$. The estimated error on corrected depth was $0.09 \mathrm{~m}$. The seabed in this area is generally flat with the $\overline{A B}$ inclination of ca. $2.3^{\circ}$ and mean depth of $5.5 \mathrm{~m}$, while the $\overline{C D}$ inclination is ca. $2^{\circ}$ with a mean depth of $5 \mathrm{~m}$, becoming deeper towards the entrance of the harbour $(\bar{D})$, with ca. $9 \mathrm{~m}$ of depth (Figure 7).

The seabed inspections using the MBS revealed the existence of three artefacts of $0.4 \mathrm{~m}^{2}$ to $2.3 \mathrm{~m}^{2}$ of area, large enough to pose risk for navigation. Artefact 1 measured ca. $0.7 \mathrm{~m} \mathrm{x}$ $0.6 \mathrm{~m}$, artefact 2 measured $0.8 \mathrm{~m} \times 0.5 \mathrm{~m}$ and artifact 3 measured $1.5 \mathrm{~m} \times 1.5 \mathrm{~m}$ (Figure 8). Artefact 1 and 2 could be metallic barrels, artefact 3 could be upside down object such as a table or a ladder. The dock wall of side $\bar{C}$ was covered by metal slabs, which were also visible 
on the acoustic images and indicated as 4 in Figure 8 . The seabed appears to be made of a top layer of soft substrate which is the general characteristics of the seabed in this area.

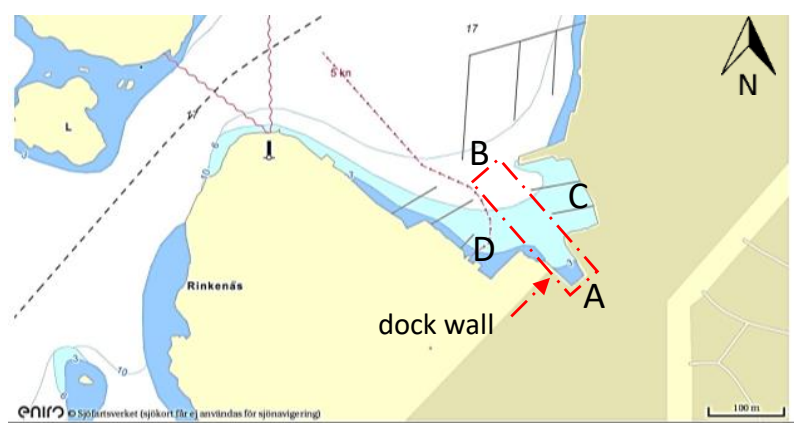

Figure 6. Overview of the surveyed area marked with red dashed rectangle on the surveyed area of a harbour located in Lysekil, $\left(58.275038^{\circ} \mathrm{N}, 11.424705^{\circ} \mathrm{E}\right)$ near a WEC factory [19].
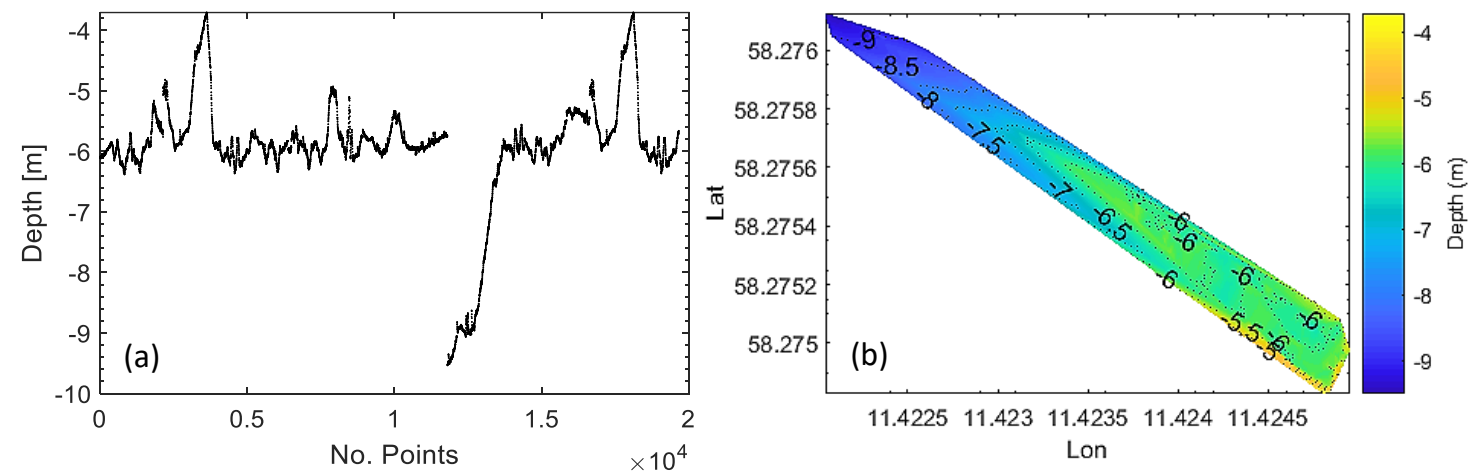

Figure 7. Ultra-high resolution bathymetry data acquired in the surveyed area $\overline{A B C D}$ demarked with a red rectangle in Figure 6. (a) Raw data of all the scouting tracks, and (b) the respective bathymetric map.
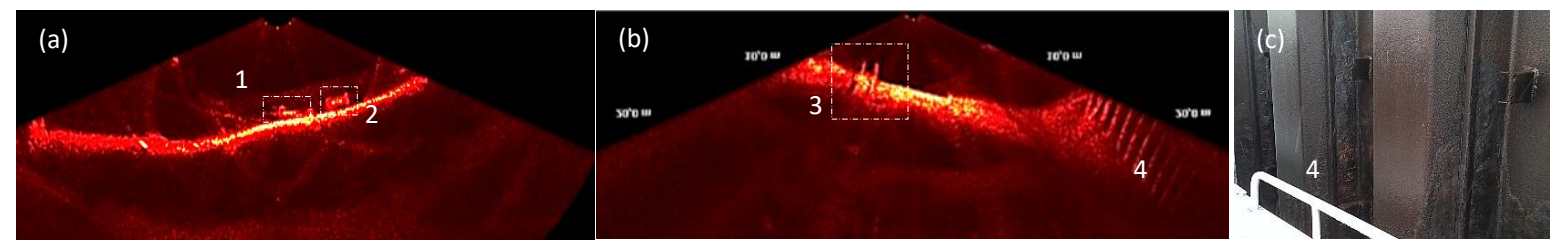

Figure 8. Overview of the seabed conditions through acoustic images $(a, b)$ showing artefacts lying on the seabed: artefact 1 measured approximately $0.7 \mathrm{~m} \times 0.6 \mathrm{~m}$, artefact 2 measured $0.8 \mathrm{~m} \times 0.5 \mathrm{~m}$ and artefact 3 measured 1.5 $\mathrm{m} \times 1.5 \mathrm{~m}$ and was located in the middle of the docking area close to the harbor steel wall indicated as 4 in both the acoustic (b) and optical images (c) and demarked with a red arrow in Figure 6.

\subsection{Example 3}

Seabed inspection and bathymetric surveys were also conducted on a proposed area for future wave power farm in Ada, Ghana (Figure 9) that supposedly will use the Seabased WEC technology [11], [19]. Here the aim was to find an area of with relatively flat seabed and bottom depths between $20 \mathrm{~m}$ to $25 \mathrm{~m}$. The surveys were conducted with a systematic parallel transects with $W_{T}$ of ca. $12 \mathrm{~m}$ to $15 \mathrm{~m}$ and the $r$ was ca. $1.55 \mathrm{~m}-1.75 \mathrm{~m}$. The uncorrected raw data referent to one of transects (Figure 10a) show measured depth values. A high resolution bathymetric map was produced (Figure 10b) with data gathered in three different surveys, and therefore the survey was a combination of systematic zig-zag and parallel transects. The optical images acquired on the surveyed site revelled that the seabed of was covered ripples of sand mixed with shell and coarse organic material (Figure 11). 


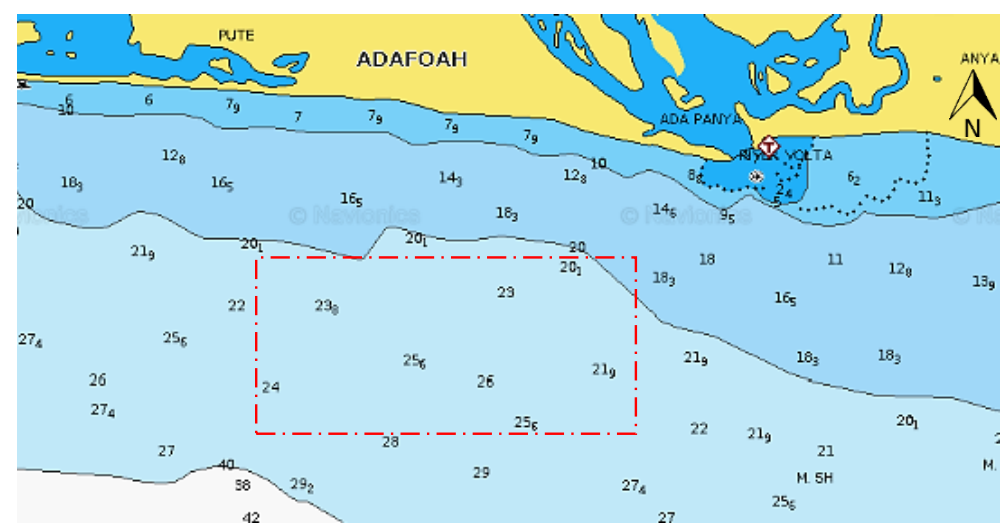

Figure 9. Nautical chart showing the bathymetry of the surveyed area (dashed rectangle) in Ada, Ghana.
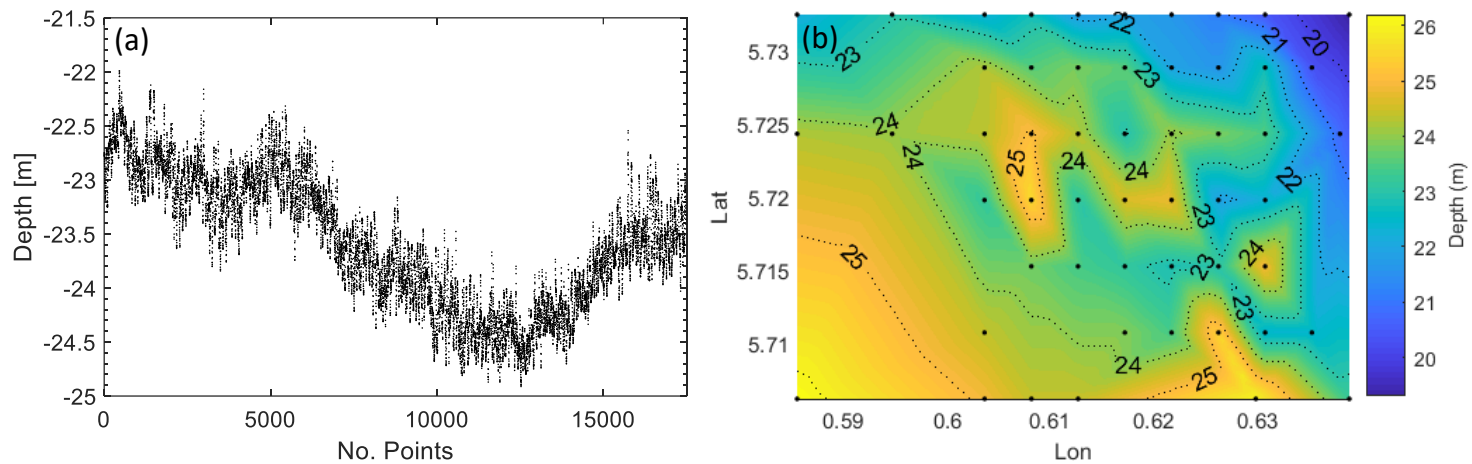

Figure 10. High resolution bathymetric of the surveyed in Ada, Ghana where a future wave farm might be located: (a) Raw data extracted from one of the scouting tracks marked by the dots in the bathymetric map (b).

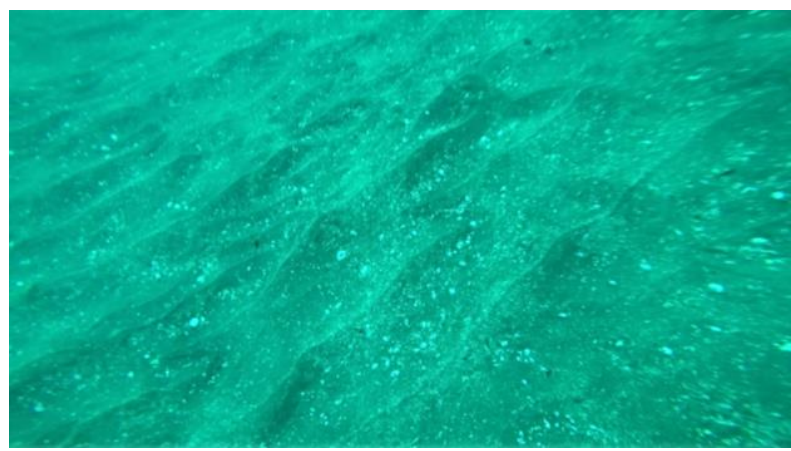

Figure 11. A clear ooptical image showing a sandy seabed with ripples of the surveyed area in Ada, Ghana.

\section{Discussion}

Both the hardware and the data processing algorithm provided good results of high resolution bathymetry as well as seabed inspections for pre-deployment of marine renewables. Although, there are several studies e.g. [14], [20]-[24] that aimed to improve the bathymetry data acquisition, this study is one of the first documented attempts to use alternatively simple and effective techniques to conduct ultra-high resolution surveys of the seabed in marine renewable energy sites. However, there is a room for improvements in terms of data acquisition, analysis and presentation. For example, the MBS can also be used for bathymetric measurements that would provide a more complete seabed mapping, which can also be combined with other existent data from satellites and nautical charts. Additional sensors such as pitch, roll, pan and tilt can be integrated to the platform in order to improve data accuracy. 
Bathymetric survey conducted in high seas can be challenging when using small vessels without dynamic positioning systems that maintain the vessel heading into a consistent track. Still, small vessels can safely be used for high resolution bathymetric data acquisition, which is the main objective of the proposed technique, as long as proper quality control protocols are conducted during and after the surveys.

\subsection{Risks associated with uneven seabed}

The greatest risk associated with the deployment of marine renewable energy devices on uneven seabed is the unsafe deployment of gravity foundations in respect to the seabed plane. External factors such as scour, bottom erosion, lateral and vertical shear forces of waves and currents can accelerate fatigue and damages to submerged devices.

Risks of incorrect deployment of devise with gravity foundation such as the Uppsala University WEC, can take three or more directions, illustrated as cases a - $\mathrm{c}$ in Figure 12. In first case, a WEC or other device with gravity foundation, lands on a tip of a slope that has curvature with a diameter significantly inferior than the diameter of a gravity foundation. In this case, it is presumed that the static equilibrium and the centre of gravity of the entire structure would be unstable so that lateral forces (red dashed arrows, Figure 12a) may cause the WEC to topple or swing. In the second case, a WEC lands in the face of a slope, here the lateral forces (dashed arrows Figure 12b) acting on the lead edge of the foundation would exert excessive loads leading to a point of rupture. If rupture occur, the gravity force can overcome the normal force so that the centre of gravity moves outside of the baseplate and the resultant force (red arrow, Figure 12b) would topple the WEC. The third case refers to a WEC that lands over two faces in between slopes or on a hole (Figure 12c). Here the resultant forces acting on the edges of the foundation would balance the gravity force acting in the centre of the foundation. Reoccurring unbalance of these forces caused by vibrations or fatigue can lead to rapture of the foundation and collapse of the WEC over its own weight. One can argue that the result movement in the second case (Figure 12b) would be the slide foundation over the seabed as the translation of the centre of gravity would naturally follow a straight line parallel to the seabed plane while the WEC tries to rotate about its centre of mass. Moreover in the case of point absorbers, this dynamic equilibrium would be compensated by the vertical forces acting on the buoy-line. However, the seabed poses friction to the foundation that might not allow it to easily slide. Lastly, vertical forces would have to be extremely high to prevent the entire WEC to collapse. 


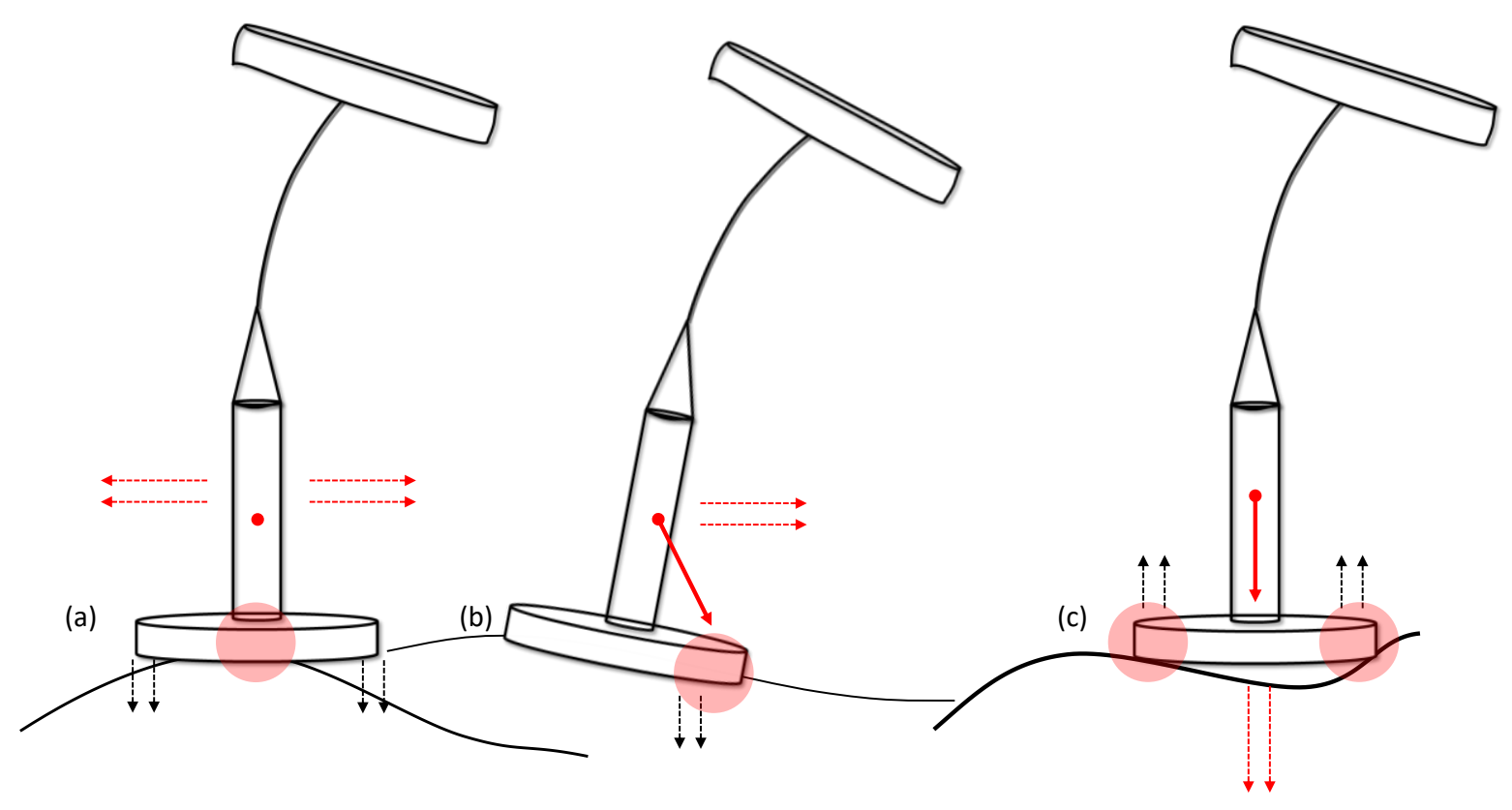

Figure 12. Three presumed scenarios that can pose risks to deployment of marine renewable energy devices using gravity foundations: (a) A device lands on tip of a slope; (b) A device lands on a face of a slope; (c) A device lands on a hole. In all scenarios the device is unstable (centre of gravity shown by red arrow), excessive loads may act on the areas indicated with red circles.

\section{Conclusions}

This study concluded that a simple subsea monitoring platform comprising a multibeam, dual beam and video cameras proved to be effective for small scale ultra-high resolution seabed inspection and bathymetric measurements. The dual beam sonar and video cameras used on this platform were ordinary and inexpensive but very precise devices. The survey time could be cut short by surveying only pre-determined sites with an assistance of an ordinary nautical chart. Measurement errors of bathymetric surveys were quantified and compensated either directly by the sonar computer or by pre-and postcalibration protocols. The main expected measurement errors were due to roll and pitch, heave, transducer draft and squat.

This study can also conclude that ultra-high resolution bathymetry data is important for a safe installation of marine renewable energy devices, principally those with gravity foundations that require a stable and flat seabed. In such cases, the spatial resolution of the bathymetry data should be at least half or a quarter of the baseplate diameter, which in most cases would require between $0.3 \mathrm{~m}$ to $2 \mathrm{~m}$ of resolution. Knowing that the marine renewable energy sector is still in an immature and fast-growing, facing tremendous technical and economic challenges that mostly affect the starting phases of a project.

\section{Acknowledgments}

This project has received funding from the European Union's Seventh Framework Programme for research, technological and demonstration under grant agreement no 607656. The authors acknowledge the Carl Trygger's and J Gust Richert's foundations for 
supporting the purchase of the split beam sonar system. The authors also would like to Acknowledge Seabased Industry AB for the collaboration on data collection in Ghana.

\section{Bibliography}

[1] R. F. Stevens and M. Saue, "Mooring Anchors for Marine Renewable Energy Foundations," in Proceedings of the 2nd Marine Energy Technology Symposium Global Marine Renewable Energy Conference April 15-17, 2014, Seattle, Washington, 2014, pp. 1-8.

[2] P. Jeffcoate and J. Mcdowell, "Performance of PLAT-I, a Floating Tidal Energy Platform for Inshore Applications," in Proceedings of the 12th European Wave and Tidal Energy Conference 27th Aug -1st Sept 2017, Cork, Ireland, 2017, pp. 18.

[3] L. J. Madjid Karimirad, Kourosh Koushan, Sam Weller, Jon Hardwick, "Applicability of offshore mooring and foundation technologies for marine renewable energy ( MRE ) device arrays," in Renew2014, International Conference on Renewable Energies OffshoreAt: Lisbon, Portugal, 2014, p. 8.

[4] S. Malhotra, "Selection, design and construction of offshore wind turbine foundations," Wind Turbines. Dr. Ibrahim Al-Bahadly (Ed.), ISBN: 978-953-307-221-0, InTech, Available from: http://www.intechopen.com/books/windturbines/selection-design-and-construction-of-offshore-wind-turbine- foundations, pp. 231-264, 2011.

[5] "Review of Options for Offshore Foundation Substructures Prepared by the Center for Wind Energy at James Madison University August 2012." James Madison University, 2012.

[6] A. Parwal, "Status Update Lysekil Research Project, Sweden," in Proceedings of the 11th European Wave and Tidal Energy Conference, 2015.

[7] M. Leijon et al., "Wave energy from the north sea: Experiences from the Lysekil research site," Surv. Geophys., vol. 29, no. 3, pp. 221-240, 2008.

[8] F. Francisco, N. Carpman, I. Dolguntseva, and J. Sundberg, "Use of Multibeam and Dual-Beam Sonar Systems to Observe Cavitating Flow Produced by Ferryboats: In a Marine Renewable Energy Perspective," J. Mar. Sci. Eng., vol. 5, pp. 1-15, 2017.

[9] F. Francisco and J. Sundberg, "Sonar for Environmental Monitoring: Configuration of a multifunctional active acoustics platform applied for marine renewables," Working manuscript, Uppsala.

[10] J. Simmonds and D. MacLennan, Fisheries acoustics: Theory and practice: Second edition, 3rd ed. Cornwall: Blackwell Science Ltd, 2007.

[11] "Seabased - Ghana Project," 2018. [Online]. Available: https://www.seabased.com/ghana.

[12] A. Aglen, "Reliability of acoustic fish abundance estimates.," University of Bergen, 1989.

[13] “Manual on Hydrography," no. M-13. International Hydrographic Bureau, Monaco, p. 554, 2005.

[14] I. M. Parnum and A. N. Gavrilov, "High-frequency multibeam echo-sounder measurements of seafloor backscatter in shallow water: Part 1 - Data acquisition and processing," Underw. Technol., vol. 30, no. 1, pp. 3-12, 2011.

[15] W. J. E. Thomson, Richard E., Data Analysis Methods in Physical Oceanography, 3rd ed. Boston: Elsevier Science, 2014.

[16] "Matlab," 2018. [Online]. Available: https://se.mathworks.com/products/matlab.html.

[17] V. P. S. S.K.Jain, “Water Resources Systems Planing and Management,” Dev. Water Sci., 2003.

[18] B. Hell, "Mapping bathymetry From measurement to applications." PhD Thesis, Department of Geological Sciences, Stockholm University, Stockholm, p. 43, 2011.

[19] M. A. Chatzigiannakou, I. Dolguntseva, and M. Leijon, "Offshore Deployments of Wave Energy Converters by Seabased Industry AB," J. Mar. Sci. Eng., vol. 5, no. 2, p. 15, 2017.

[20] A. Avdis, C. Jacobs, J. Hill, G. Gorman, and M. Piggott, "Shoreline and bathymetry approximation in mesh generation for tidal renewable simulations," in Proceedings of the 11th European Wave and Tidal Energy Conference 611th Sept 2015, Nantes, France, 2015.

[21] P. Bell, "Remote detection of sea surface roughness signatures related to subsurface bathymetry, structures and tidal stream turbine wakes," in Proceedings of the 11th European Wave and Tidal Energy Conference, Nantes, France, 2015.

[22] V. E. Schmidt and Y. Rzhanov, "Measurement of Micro-bathymetry with a GOPRO Underwater Stereo Camera Pair," in Oceans, 2012.

[23] A. Mohammed, S. Sciences, M. M. A. Kotb, S. B. El Kafrawy, S. Sciences, and H. A. Monsef, "Bathymetry Mapping Using High Spatial Resolution Satellite Images," in 4th Young Researches Conference, Suez Canal University, Ismaillia, Egypt, 29th -30th April 2017.

[24] X. Monteys, P. Harris, S. Caloca, and C. Cahalane, "Spatial Prediction of Coastal Bathymetry Based on Multispectral Satellite Imagery and Multibeam Data," Remote Sens., vol. 7, pp. 13782-13806, 2015. 